\title{
Interactions zooplancton-poissons dans une retenue oligotrophe de mise en eau récente (Ste-Croix, Provence, France)
}

\section{Long-terri interactions between zooplankton and fish comrnunities in a large oligotrophic réservoir of South-East France}

\author{
D. Pont, R. Chappaz, G. Brun et A. Champeau
}

Volume 2, numéro 4, 1989

URI : https://id.erudit.org/iderudit/705054ar

DOI : https://doi.org/10.7202/705054ar

Aller au sommaire du numéro

Éditeur(s)

Université du Québec - INRS-Eau, Terre et Environnement (INRS-ETE)

ISSN

0992-7158 (imprimé)

1718-8598 (numérique)

Découvrir la revue

Citer cet article

Pont, D., Chappaz, R., Brun, G. \& Champeau, A. (1989). Interactions zooplancton-poissons dans une retenue oligotrophe de mise en eau récente (Ste-Croix, Provence, France). Revue des sciences de l'eau / Journal of Water Science, 2(4), 777-792. https://doi.org/10.7202/705054ar

\section{Résumé de l'article}

Les peuplements zooplanctoniques et piscicoles de la retenue de Sainte-Croix (lac oligotrophe profond) ont été suivis de la mise en eau (1974) à 1986.

La phase initiale de colonisation (1974-1975), caractérisée par le calanide Acanthodiaptomus denticornis et la dominance des espèces piscicoles peuplant antérieurement le Verdon lotique (Barbus fluvatilis, Leuciscus cephalus) a été de courte durée. Par la suite, les cladocères sont restés dominants avec cependant de profondes modifications dans la structure du peuplement. En 1977-78, Daphnia longispina était dominante tout au long de l'année (76 \%). Plus récemment, cette espèce ne représentait plus que $34 \%$. des effectifs (1983-84), puis $24 \%$ (1985-1986). La situation actuelle est caractérisée par la présence de Diaphanosoma brachyurum en été et l'abondance des petites formes (bosmines).

L'ichtyofaune s'est elle aussi modifiée avec, notamment, une importante population d'ablette (Alburnus cluburnus), exploitant la zone pélagique. Son régime alimentaire est largement zooplanctonophage mais inclut mollusques et insectes lorsqu'elle est capturée en zone littorale.

Cette augmentation de la pression de prédation a entraîné un net déplacement du spectre des tailles du zooplancton vers tes classes inférieures à $0,8 \mathrm{~mm}$ en 1986 (82 \%) alors que les grandes formes ( > à $1 \mathrm{~mm}$ ) représentaient $60 \%$ des effectifs en 1977-78. Les densités de $D$. longispina et $D$. brachyurum décroissent rapidement entre 1983 et 1986.

Les conséquences de cette prédation sélective sont discutées (évolution prévisible du système) ainsi que les causes pouvant expliquer l'absence de régulation efficace (densité-dépendance) dans un tel système prédateur-proie.
Ce document est protégé par la loi sur le droit d'auteur. L’utilisation des services d’Érudit (y compris la reproduction) est assujettie à sa politique d'utilisation que vous pouvez consulter en ligne.

https://apropos.erudit.org/fr/usagers/politique-dutilisation/ 


\title{
Interactions zooplancton-poissons dans une retenue oligotrophe de mise en eau récente (Ste-Croix, Provence, France)
}

\author{
Long-term interactions between zooplankton and fish communities \\ in a large oligotrophic reservoir of South-East France
}

D. PONT(1), R. CHAPPAZ (2), G. BRUN(2), A. CHASAPEAU(2)

RÉSUMÉ

\begin{abstract}
Les peuplements zooplanctoniques et piscicoles de la retenue de Sainte-Croix (lac oligotrophe profond) ont été suivis de la mise en eau (1974) à 1986.

La phase initiale de colonisation (1974-1975), caractérisée par le calanide Acanthodiaptomus denticornis et la dominance des espèces piscicoles peuplant antérieurement le verdon lotique (Barbus bluviatilis, Leuciscus cephalus) a été de courte durée. Par la suite, les cladocères sont restés dominants avec cependant de profondes modifications dans la structure du peuplement. En 1977-78, Daphnia longispina était dominante tout au long de l'année ( $76 \%$ ). Plus récemment, cette espèce ne représentait plus que $34 \%$ des effectifs $(1983-84)$, puis $24 \%$ (1985-1986). La situation actuelle est caractérisée par la présence de Diaphanosoma brachyurum en été et l'abondance des petites formes (bosmines).
\end{abstract}

L'ichtyofaune s'est elle aussi modifiée avec, notamment, une importante population d'ablette (Alburnus clburnus), exploitant

(1) Departement de Biologie Animale et Ecologie. U.A. CNRS $n^{\circ} 68$, Université Claude Bernard (Lyon I), 43, boulevard du 11 novembre 1918, 68622 Villeurbanne Cedex, France

(2) Laboratoire d'Hydrobiologie, Université de Provence, 1, place Victor Hugo, 13331 Marseille Cedex 3, France. 
La zone pélagique. Son régime alimentaire est largement zooplanı tonophage mais inclut mollusques et insectes lorsqu'elle est capturée en zone littorale.

Cette augmentation de la pression de prédation a entrainé un net déplacement du spectre des tailles du zooplancton vers les classes inférieures à $0,8 \mathrm{~mm}$ en $1986(82 \%)$ alors que les grandes formes ( $>$ à $1 \mathrm{~mm}$ ) representaient $60 \%$ des effectifs en 1977-78. Les densités de 0 . longispinc et 0 . brachyurum décroissent rapidement entre 1983 et 1986.

Les conséquences de cette prédation sélective sont discutées (évolution prévisible du système) ainsi que les causes pouvant expliquer l'absence de régulation efficace (densité-dépendance) dans un tel système prédateur-proie.

Mots clés : Rëservoir, zooplancton, prédation sélective, daphnia longispina, alburnus alburnus.

\section{SUMMARY}

The aim of this study was to identify relationships between changes that occurred, from 1974 to 1986, both in the limnetic zooplankton and the fish communities of the large reservoir of Sainte-Croix (Southern France).

This reservoir (figure 1 ) is characterized by a capacity of $767 \mathrm{hm}^{3}$ and a long average renewal period (280 days). It was filled up in 1974. Changes in levels can attain $16 \mathrm{~m}$, especially in winter. During summer, the lake is clearly stratified : surface water temperatures are about $22-250^{\circ} \mathrm{C}$ whereas waters below the thermoctine are cooler $\left(<10^{\circ} \mathrm{C}\right)$ but well oxygenated (more than $50 \%$ saturation). According to the mean annual total phosphorus $\left(19 \mathrm{mg} / \mathrm{m}^{3}\right)$ and chlorophyll a (ranging from 0.5 to $2 \mathrm{mg} / \mathrm{m}^{3}$ ), the lake is oligotrophic.

The zooplankton was collected with a Clarke-Bumpus devise sampler (125 $\mu \mathrm{m}$ mesh-size) during both the 1974-1978 and the 1983-1984 periods. More recently (1985-1986), vertical hauls of zooplnakton were made with a $50 \mu m$ mesh-size aperture net. Only relative abundance of large species (excluding small rotifers) have been taken into account for comparisons between. different periods (figure 2). For the last four years (1983-1986), a set of data on fittration efficiency and comparison between samplinc methods provides information concerning the evolution of annual mean densities of zooplankton species (figure 3 ).

Successive inventories of the fish species were made in 1976 , 1977, 1984 and 1987 at the same Location with $15 \mathrm{~mm}, 27 \mathrm{~mm}$ and $50 \mathrm{~mm}$ mesh-size nets respectively. Figure 1 shows that abundant autochtonous species (Chondrostoma chondrostoma, Barbus fluviatilis/during the first period (1976-1977) are now relatively scarce, whereas the roach (Rutilus rutilus), an introduced species, is now largely dominant. Likewise, the relative abundance of the bleak, Alburrus alburnus, also increased, especially during the last period (1984-1987). Only this last species was caught by fine mesh-size nets in the pelagic epilimnion. Adults fed mostly on zooplankton but also took more molluscs in the littoral area (stomach analysis). 
In 1974, one year after an overflow, the calanoid Acanthodiaptomus denticornis represented the majority of zooplankton collected but it disappeared quickly. Since 1976, cladocerans are largely dominant. General evolutive trends show a decrease in relative abundance of Daphnia longispina from 1978 to 1986 whereas Diaphanosoma brachyurum have been recorded since 1983. Quantitative data (figure 3 ), available for the last 4 years (1983-1986) show that the population densities of both these two large cladoceran and cyclopoid species (mainly Macrocyclops albidus) declined radically. On the other hand, small species such as Bosmina longirosiris. Bosmina coregoni and Asplanchna priodonta exhibited the same population densities throughout the last period. Comparisons of phytoplankton community structure from 1982 to 1986 do not show signicative trends. Thus, variations in resource abundance cannot explain the long-term evolution of zooplankton communities.

By comparing the mean annual length frequency distributions of zooplankton in 1977-78 and 1986 (figure 4), a marked decline of large bodied forms is seen. The individuals larger than $1.0 \mathrm{~mm}$ represented $60 \%$ of total organisms in 1977-73 against $14.5 \%$ in 1986. Mean annual lenghts were $0.8 \mathrm{~mm}$ in 1977-78 and $0.3 \mathrm{~mm}$ in 1986. These results are in good agreement with BROOKS and DODSON hypothesis that planktivorous $f$ ish can have a profound effect on zooplankton community taxonomics and size structure. They show that the control of structure and abundance of zooplankton by fish predation in new man-made lakes is a reality.

Most fish are general predators. As demonstrated by MURDOCH and BENCE (1987), this can explain the instability of the coupled predator-prey system in the pelagic portion of SainteCroix reservoir because of : i) the capacity of the bleak to use fat reserves and to change its diet when large zooplankton prey density is too low, $i i$ ) the generation time of the predator is much longer than that of planktonic preys. Thus, the classical density-dependence mechanisms do not stabilize the predator-prey system and the main planktonic prey tend to disappear (D. longispina, in our case).

Nevertheless, some potentially stabilizing mechanisms could interfere in the system before the local extinction of the prey : compensatory responses by the prey and significant changes in the fish community. There is reason to consider : 1) The existence of refuges in space. Vertical size distribu$t i$ on of $D$. Longispina (unpublished data) show that there is a strong vertical size gradient during daytight hours. Larger-bodied reproductive forms (Longer than $1 \mathrm{~mm}$ ) are only present in the deeper layers $(20-30 \mathrm{~m})$ whereas, during the night all the organisms migrate to the superficial layer.

2) The existence of refuge in time. In the past (1976-1978), D. longispina was dominant all year around. More recently (4983-1986), population densities were high only in spring (figure 5). From april to june, bleaks tend to assemble in the littoral zone for reproduction.

3) An increase in the bleak mortality rate due to a recent development of large predator populations, such as Esox esox, could permit a reduction or, at least, a stabilization of the bleak population growth rate. 
Key-words : Resevoir, zooplankton, selective predation, daphnia longispina, bleak.

INTRODUCTION

Les interactions entre zooplancton et poissons s'intègrent dans le cadre plus général des relations prédateurs proies au sein des systèmes limniques. Bien que les poissons ne regroupent pas à eux seuls la totalité des consomateurs secondaires susceptibles d'exploiter le zooplancton, il reste néanmoins que la composition du peuplement ichtyologique est, tout comme la production primaire, un facteur déterminant agissant sur la structure du peuplement zooplanctonique (POURRIOT, 1982).

Les conséquences directes de cette prédation ont pu être mises en évidence tant pour la composition spécifique du peuplement zooplanctonique que pour la réduction de la taille moyenne des organismes par prédation sélective (HRBACEK et al., 1961 ; BROOKS et DODSON, 1965 ; IAZZARO, 1987 ; O'BRIEN, 1975). L'efficacité de capture et le mode de sélection des proies est largement fonction, d'une part, de la taille et du comportement alimentaire des poissons (JANSSEN, 1980 ; COOPER et al., 1985 ; O'BRIEN, 1987), et d'autre paxt de la visibilité et de la vitesse d'échappenent de la proie (O'BRIEN, 1979 ; KERFOOT et al., 1980). A terme, les proies développent des stratégies adaptatives tendant à minimiser les effets de cette prédation : migrations verticales, polymorphisme, cyclomorphose (HUTCHINSON, 1967 ; ZARET et SUFFERN, 1976 ; POURRIOT, 1983).

L'étude des milieux nouvellement crêes pax l'Home se prête particulièrement bien à l'analyse de l'évolution $a^{\prime} u n$ tel système interactif comune l'ont montré les travaux menés sur des étangs piscicoles ou des lacs de barrage (GRYGIEREK et $\alpha l ., 1966$; BALVAY, 1985).

La retenue hydroélectrique de Sainte-croix a fait l'objet d'un important suivi, depuis sa mise en eau en 1973, de la production primaire et des peuplenents zooplanctonique et ichtyologique (C.T.G.R.E.F., 1977 ; CHAMPEAU et al., 1979 ; GREGOIRE, 1982 ; CHAMPEAU et al., 1982 ; BRESSAC et CHAMPEAU, 1985 ; CHAPPAZ, 1986 ; AMRANI, 1987 ; BIN-MOLE, 1987 ; EL HAFA, 1987).

L'objectif de cet article est d'analyser les conséquences de l'évolution du peuplement ichtyologique sur la composition et la dynamique du zooplancton au cours des treize premières années de rise en eau de la retenue et de montrer 1 'importance de la prédation comne facteux-clé du processus évolutif par rapport aux différents paramètres les plus courament cités dans ce type d'écosystème (BALVAY, 1985) : maturation progressive du système, évolution du niveau trophique par modifications des apports en provenance du bassin versant, narnage.... Les évolutions possibles à moyen terme des deux compartiments, zooplanctonique et ichtyologique, seront enfin discutêes à la lumière des tendances mises en évidence au cours de ces cinq dernières années. 
MATÉRIEL ET METHODES

La retenue de Sainte-Croix est située à la limite des départements du var et de Haute-Provence. Elle s'insère en troisième position dans un complexe hydro-électrique de cinq barrages implantés le long du cours du verdon. C'est 1 'un des plus grands plans d'eau artificiels de France avec une superficie de 2182 ha $(12 \mathrm{~km}$ de long pour $2 \mathrm{~km}$ de large en moyenne), une capacité de $767 \mathrm{hm}^{3}$ pour un bassin versant de $1591 \mathrm{~km}^{2}$. La profondeur augmente rëgulièrement de l'amont vers l'aval, passant de $10 \mathrm{~m}$ à plus de $80 \mathrm{~m}$ à l'aplomb du barrage. Le temps de renouvellement théorique des eaux est de 200 jouxs. Le marnage est de type saisonnier avec une amplitude hivernale maximale de $16 \mathrm{~m}$.

C'est un lac monomictique chaud (selon HUTCHINSON, 1967). L'influence méditerranéenne se traduit par des températures minimales hivernales voisines de $6^{\circ} \mathrm{C}$ et une période de réchauffement des eaux (activité biologique) très longue par rapport aux milieux de ce type situés plus au Nord (CASPER, 1985) : d'avril à novembre avec une përiode de stratification sensus stricto s'étendant de fin juin à mi-octobre. La thermocline s'enfonce progressivement durant cette dernière période, de 7-10 m à 15-20 m. L'épilimnion se caractérise par des températures variant entre 21 et $24^{\circ} \mathrm{C}$.

Les prélèvements zooplanctoniques réalisés de 1974 à 1978 (GREGOIRE, 1982 ; CHAMPEAU et al., 1982) ont été réexaminés et interprétés en ne considérant que les abondances relatives (échantillonnage de type seniquantitatif).

En 1985 et 1986, des traits verticaux fond-surface ont été effectués à plusieurs stations situées le long du gradient amont-aval (AMRANI, 1987) selon une périođicité mensuelle (1985) ou hebdomadaire à bi-mensuelle (1986). L'engin de capture consistait en un filet caractérisé par un vide de maille de $50 \mu \mathrm{m}$ et un rapport de surface ouverte de 7,3 . Ce rapport se définit par le quotient de la surface à 1 'embouchure et de la surface effective de sortie de l'eau. Des valeurs supérieures a 3 sont classiquement considérées comne satisfaisantes (LAMOTTE et BOURLIERE, 1971). Les influénces de la saisonnalité et de l'usure du filet sur l'efficlence de filtration ont par ailleurs été analysées et les résultats corrigés en conséquence.

Les densités de zooplancton obtenues pour la période juin 1982 à fin 1984 (prélèvenent à I'échantillonneur Clarke-Bumpus) ont été calculêes d'après BRESSAC (1985). Six séries de tests ont permis de comparer l'efficacitê de cet échantillonneur avec celle du filet vertical utilisé à partir de 1985. Les densitês obtenues à l'aide du Clarke Bumpus doivent être multipliées par un facteur moyen de $8,7(S x=1.36)$.

Toutes les densitês mensuelles par $\mathrm{m}^{3}$ présentées pour la période 1982 à 1986 représentent une moyenne des différents échantillons récoltês sur 1 'ensemble du gradient amont-aval đurant la période considérée $(5<n<64)$.

L'analyse de l'évolution du peuplenent ichtyologique est basée sur les pêches réalisées en 1976 par le C.T.G.R.E.F. (1977) et en 1984 par ChAPPAZ (1986) dans la zone littorale médiane du lac (baie des Salles) durant ia përiode de stratification, a l'aide de filets maillants de 
$15 \mathrm{~mm}, 27 \mathrm{~mm}$ et $50 \mathrm{~mm}$. Une série de pêche a été effectuée en octobre 1987 dans la baie des Salles à l'aide de moyens de capture identiques. Le total des prises s'élève respectivenent à 107,142 et 19 individus pour les filets maillants de 15, 27 et $50 \mathrm{~mm}$. Seules les abondances relatives des espèces récoltêes à l'aide d'un même type de filet sont comparables entre elles. Ces résultats sont par ailleurs corroborés par l'ensemble des pêches réalisées en zone littorale de 1983 à 1985 (CHAPPAZ, 1986).

De juin 1987 à mai 1988, des filets maillants de $14 \mathrm{~mm}$ de vide de maille et de longueur 30 à $40 \mathrm{~m}$ ont été posés dans la zone pélagique en surface afin d'apprécier sa fréquentation par les poissons (effectif total capturé de 499 poissons en 8 pêches).

RESULTATS

1 - Le peuplement ichtyologique (figure 1)

En 1976, moins de trois ans après la mise en eau complète de la retenue, les espèces caractéristiques du verdon lotique sont encore bien reprësentêes: Chevaine (Leuciscus cephalus), Toxostome (Chondrostome toxostomal.... D'autres espèces issues d'empoissonnement apparaissent : Perche (Perca fluviatilis), Gardon (Rutilus rutilus), Tanche (Tinca tinca).... Les captures réalisées en 1977 (CHAMPEAU et al., 1979) confirment en particulier la rareté des ablettes'et des gardons à cette période.

En 1984, les espèces introduites dominent le peuplement avec l'Ablette (Alburnus alburnus), le Goujon (Gobio gobio) et le Gardon. L'examen des pêches de 1987 ne fait que confirmer cette tendance en l'amplifiant. On assiste en effet à une raréfaction des toxostomes et des chevaines dans les prises réalisées avec les filets de maille $15 \mathrm{~mm}$ et 27 run. L'ablette représente plus de 80 \& des captures dans des filets de maille $15 \mathrm{~mm}$ et le gardon plus de 85 \% dans les filets de $27 \mathrm{mrn}$.

Malgré I'importance de 1 'empoissonnement, les truites n'ont pas pris au fil des ans une place notable au sein du peuplement. A l'inverse, le développement des populations d'ablettes est remarquable. Son origine semble résider, d'une part, dans le développement des populations autochtones présentes dans le verdon avant la création du barrage et, d'autre part, dans les déversements initiaux de poissons réalisés dans la retenue en 1975-1976: quelques dizaines de kilos d'ablettes (source: A.P.P. du Var).

L'ensemble des campagnes de pêche réalisées en 1987 montre que 1 'ablette est le seul poisson occupant de façon rểellement importante la zone pëlagique (en moyenne plus de 95 des captures). Ont été également récoltês quelques goujons, brochets et truites.

L'analyse des rêgimes alimentaires des espèces dominantes par CHAPPAZ (1986 : examen des contenus stomacaux) montre que seules deux espèces peuvent être considérées comme susceptibles d'exercer une prëdation importante sux les populations de microcrustacés. 

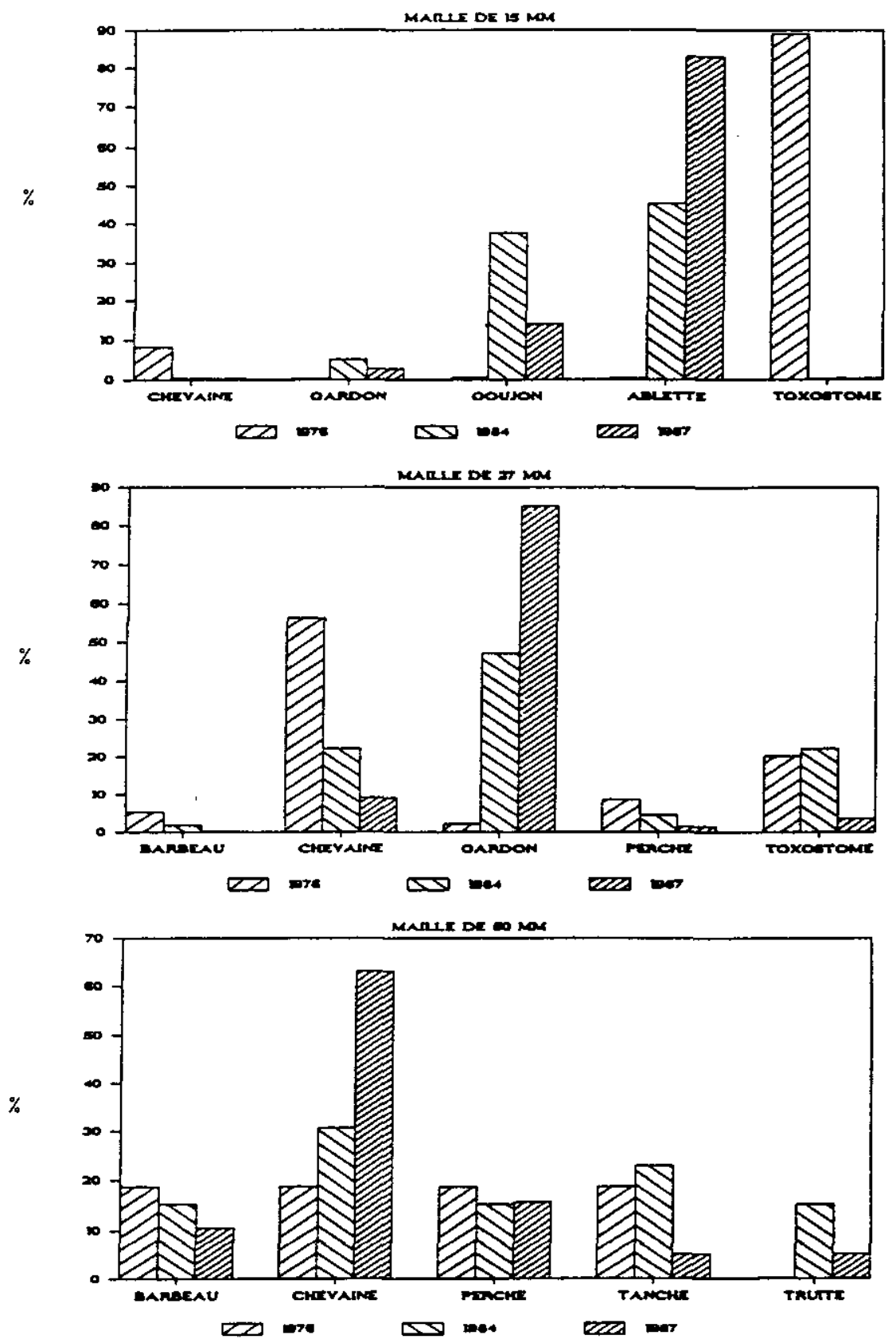

Figure 1. - Evolution de la structure du peuplement ichtyologique de 1976 à 1987 estimées d'après les nombres de captures réalisées en zone littorale à l'aide de filets maillants de 15, 27 et $50 \mathrm{~mm}$. (Données brutes de 1976 extraites du rapport C.T.G.R.E.F., 1977).

Figure 1. - Percent frequency histograms comparing the frequency distributions of species (densities) from 1976 to 1987, using 3 mesh-size nets $(15,27$ and $50 \mathrm{~mm})$ in littoral area. 
Cyclopides et daphnies représentent 56 du nombre total de proies observées dans les contenus stomacaux de stades immatures de perche (4 à $10 \mathrm{~cm}$ ) mais seulement 1,2 des biomasses ingérées en raison de leur comportement ichtyophage. Les espèces zooplanctoniques ingérées sont de grande taille (Sida cristallina, Simocephalus vetulus, Macrocyclops albidusl et habituellement rencontrées dans les zones littorales et les herbiers. Ces proies ne sont pas observées en zone pélagique à I'exception de Macrocyclops albidus.

L'analyse des contenus stomacaux d'ablettes pêchées dans la zone centrale de la retenue montre que le zooplancton est le type de proie le plus fréquent : occurrence et fréquence moyennne respectivement de 88 \& et 63 \& (CHAPPAz et al., 1987). L'analyse de ces tractus digestifs (en cours) indique une forte ingestion des espèces dominantes de la zone pélagique et, en particulier, D. longispina.

En définitive, le peuplement ichtyologique de la retenue de Saintecroix s'est profondément modifiè au cours des quatorze premières années qui ont suivi la mise en eau. On assiste en particulier au développement d'une importante population d'ablettes zooplanctonophages capables d'exploiter les espèces présentes dans la zone pélagique du lac.

\section{2 - Le peuplement zooplanctonique (figure 2)}

Un an après le début de la mise en eau (1974), le peuplement zooplanctonique est très largement dominé par la calanide Acanthodiaptomus denticornis (93 des effectifs). Cette espèce, présente dans les retenues situées en amont (GREGOIRE, 1982), est abondante dans d'autres lacs de barrage du Sud-Est de la France (BRESSAC, 1983). Dès 1975, l'abondance relative de ce copépode décrồt $(27$ \&) pour n'être plus qu'un êlément secondaire de cette zoocénose (noins de 5 ) en 1977-1978. Cette espèce disparaît pratiquement de la masse d'eau à partir de 1978. Suite à cette courte période de colonisation, les cladocères dominent le peuplement.

De 1975 à 1978, Daphnia longispina est dominante tout au long du cycle annuel (en moyenne 69 \&), accompagné de Ceriodaphnia pulchella (11 \&) en automne et, secondairement, du genre Bosmina ( 8 \&).

De 1983 à 1986, le cortège faunistique est comparable avec, cependant, une structure différente. $D$. Longispina ne représente plus que 33 des organismes et ne reste dominante que durant la période printanière avec un pic d'abondance à la fin mai (AMRANI, 1987). Deux espèces voient leur abondance relative augmentex = C. pulchella (21 8) en automne et les deux bosmines $B$. Rongirostris et $B$. coregoni $(26)$, au printenps et lors de la période de mélange. La modification essentielle réside néanmoins dans l'apparition de Diaphanosoma brachyurum. Espèce estivale typique, elle se développe tous les ans en fin d'été (figure 5 ) et reste abondante durant 2 mois environ (aoūt-septembre).

Les cyclopides sont représentês essentiellement en zone pélagique par Cyclops vicinus vicinus et Macrocyclops albidus. Ces deux espèces, dont le régime alimentaire est le plus souvent carnivore (FRYER, 1957), ne représentent que 5 à 6 \& de I'effectif total depuis 1977. L'accroissement relatif de $M$. albidus au détriment de $C$. $v$. vicinus au cours $d \in s$ dix dernières années pourrait ètre lié au dêveloppement $d$ 'un important herbier à Potamageton et Myriophyllum dans la zone littorale. 


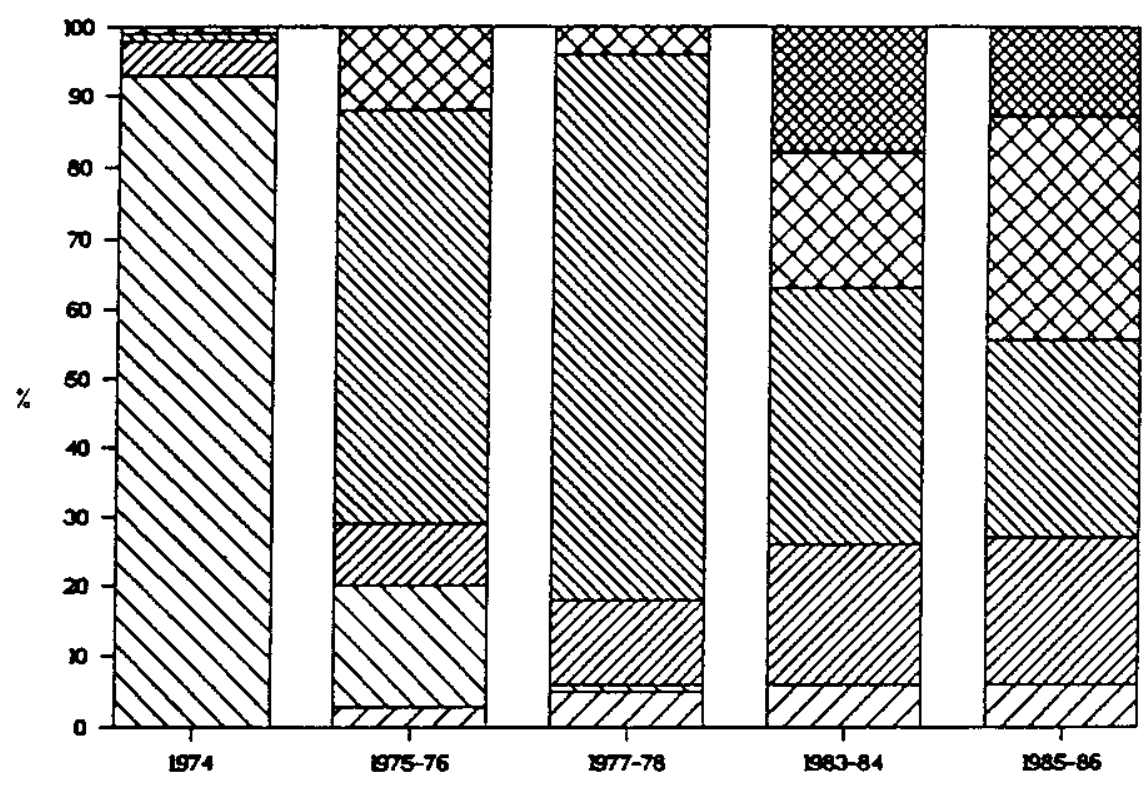

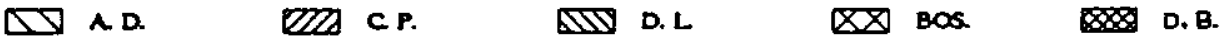

ZCYc.

Figure 2. - Evolution de la structure moyenne annuelle (en $z$ ) du peuplement zooplanctonique (copépodes et cladocères) de la mise en eau à 1986 .

Figure 2. - Relative mean annual abundances (in percent) of copepod and cladoceran species from 1974 (after flood) to 1986 .

\author{
A.D. : Acanthodiaptomus denticornis \\ D.L. : Daphnia longispina \\ D.B. : Diaphanosoma brachyurum \\ C.P. : Ceriodaphnia pulchella \\ CVC : Cyclopides IMacrocyclops albidus + \\ Cyclops vicinus vicinus \\ BOS : Bosmina langirostris + Bosmina coregoni
}

Les caractéristiques des engins de captures utilisés de 1974 à 1978 n'ont pas permis d'échantillonner la plupart des rotifères. Les prélèvements de 1986 montrent que ces organismes sont bien représentés à 1 'heure actuelle, dans la zone pélagique (au moins neuf espèces, 48 \& des densités totales moyennes) avec notamment Kellicotia longispina, Polyarthra vulgaris, Synchaeta sp.. L'espèce de grande taille Asplanchna priodonta est le seul représentant de ce groupe pour lequel l'effort d'échantillonnage semble avoir été suffisant depuis la mise en eau. Son abondance relative s'est accrue régulièrement en regard du nombre total de microcrustacés : 0,5 en 1975-1976, 2 en 1977-1978, 17 \& de 1983 à 1986.

La comparaison des deux périodes les plus récentes (1983-1984 et 1985-1986) montre que le peuplement de cyclopides et de cladocères n'a 
pas encore atteint un stade d'équilibre. L'abondance relative de 0 . longispina continue de décroitre (de 37 à 29 \%). La situation de D. brachyurum est comparable $(18$ \& a 13 \%). La part de C. pulchella reste stable tandis que les deux espèces de bosmines sont naintenant dominantes (de 19 à 32 ).

L'examen de l'évolution des densités moyennes annuelles confirme ce résultat (figure 3). On observe également une réduction d'environ 50 \% des effectifs de $D$. longispina et $D$. brachyurum entre les périodes 19831984 et 1985-1986 (respectivement de 4400 à 2300 et de 2100 a 1000 ind.) $\mathrm{m}^{3}$ ). Les densités de $C$. pulchella diminuent égalenent bien que plus modérément (respectivement de $2400 \mathrm{a} 1600 / \mathrm{m}^{3}$ ). Par contre, les effectifs de C. pulchella restent stables (respectivement 2500 à $2600 / \mathrm{m}^{3}$ ).

Les changements les plus récents (1983 à 1986) ne semblent pas liês à une modification globale du fonctionnement du système, puisque l'on n'observe pas d'accroissement des densités des espèces de petite taille (bosmines). Seuls les effectifs des deux especes de plus grande taille diminuent.

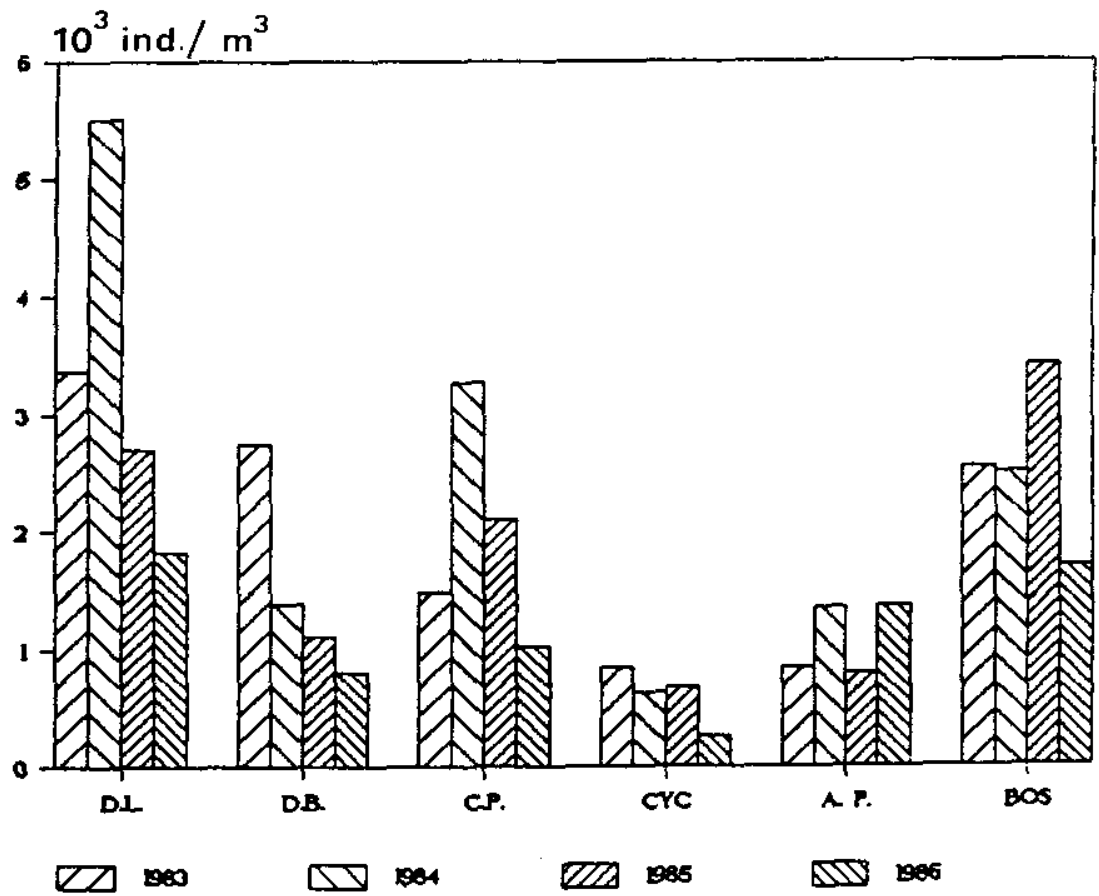

Figure 3. - Evolution des densités annuelles moyennes $110^{3}$ ind. $/ \mathrm{m}^{3}$ ) des différentes espèces macrozooplanctoniques de 1983 à 1986.

Figure 3. - Mean annual density $\left(10^{3}\right.$ ind. $\left./ \mathrm{m}^{3}\right)$ of macrozooplankton species from 1983 to 1986.

D.L. : Daphnic longispina

D.B. : Diaphanosoma brachyurum

C.P. : Ceriodaphnia pulchella

CVC : Cyclopides (Machocyclops albidus + Cyclops vicinus vicinus)

A.P. : Asplanchna priodonta

BOS : Bosmina longirostris + Bosmina coregoni 
La comparaison des distributions moyennes annuelles des fréquences de taille des copépodes et cladocères en 1977-1978 et 1986 (figure 4) met clairement en évidence un net déplacement du spectre vers les classes de taille inférieure. En 1977-1978, 60 des organismes ont une taille supérieure à $1,0 \mathrm{~mm}$ contre 14.5 en 1986 (D. longispina, M. albidus). on note la quasi disparition des organismes de taille supérieure à 1,7 mun alors que ceux-ci représentaient 2,5 en 1977-1978. Actuellement, le peuplement est largement doniné par des individus de taille voisine du demi-millimètre.

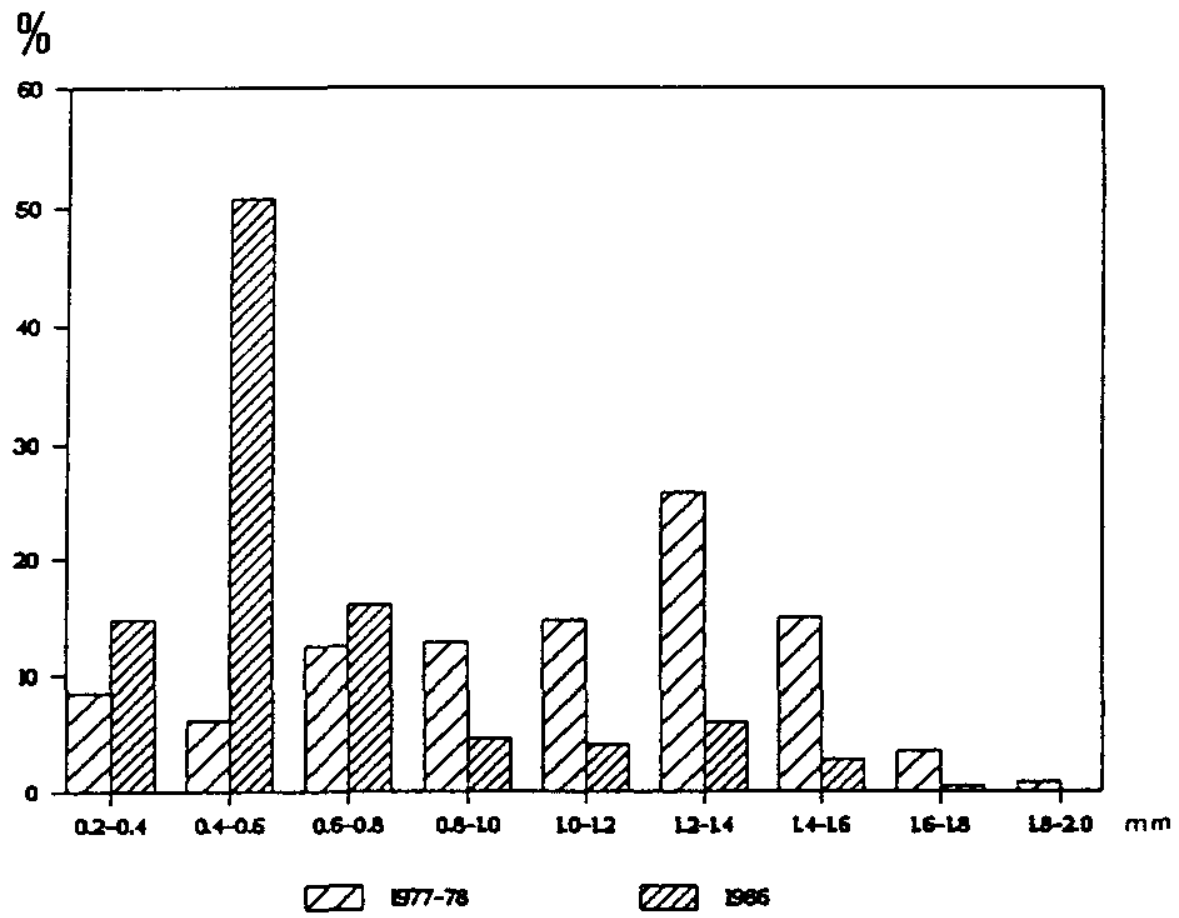

Figure 4. - Comparaison des structures de taille du macrozooplancton (copépodes + cladocères + Asplanchna priodonta) en 1977-1978 et 1986 (fréquence en \& par classe de taille d'amplitude $0,2 \mathrm{~mm})$.

Figure 4. - Comparison of mean annual length frequency distributions of zooplankton (Copepods + Cladocera + Asplanchna priodonta) in 1977-1978 and 1986 (as expressed in percent).

\section{DISCUSSION}

Au cours de l'évolution d'une retenue après la phase initiale de mise en eau, trois étapes ont été classiquement décrites (BALVAY, 1985) : explosion trophique, dépression trophique et stabilisation. 
Dans le cas de la retenue de Sainte-Croix, la production primaire durant les trois premières années de fonctionnement était relativement plus forte qu'actuellement (CHAMPEAU et al., 1982 ; GREGorRE, 1982 ; EI HAFA, 1987). De même, la teneur moyenne en orthophosphates s'élevait a $70 \mu g / 1$ (CHAMPEAu et $a l$., 1979). Ce bloom trophique est cependant resté d'ampleur modeste en raison de la faible disponibilité en éléments nutritifs dans les terrains nouvellement inondés (très peu d'activité agricole). Les bionasses phytoplanctoniques maximales, exprimées en chlorophylle " $a$ ", sont restées inférieures à $5 \mathrm{mg}$ de chlor. " $\alpha$ " $/ \mathrm{m}^{3}$ (1976) et ont diminué de moitié dès 1977. Du point de vue zooplanctonique, cette période a vu, dès 1975, le cladocère D. Longispina succéder au calanide Acanthodiaptomus denticornis. Le développement de cette dernière espèce caractérise donc clairement la phase initiale de colonisation. Les valeurs des coefficients de condition des espèces de poisson dominantes en 1977 (chevaine, toxostone) sont significativenent plus élevées que celles mesurées sur les populations des retenues plus anciennes situées en aval (CHAMPEAu et al., 1979).

Suite à cette première phase, la retenue a évolué rapidement vers un êtat d'oligotrophie prononcé qui la caractérise actuellement, en raison de la faiblesse des apports en provenance de la zone amont (relachûre des barrages en aval) et de la pauvreté du bassin versant immédiat (EL HAFA, 1987) : orthophosphates indécelables (< à $1 \mu \mathrm{g} / 1),(\mathrm{P}$ total) moy $=19 \mu \mathrm{g} / 1,(\operatorname{chlor} . a)=\mathrm{de} 0,5$ à $2,5 \mu \mathrm{g} / 1$.

L'examen de la composition et de la structure du peuplement zooplanctonique de 1977-1978 à 1986 semble clairement indiquer que 1'evolution de ce dernier est beaucoup plus dépendante des modifications survenues au sein du peuplement ichtyologique que de variations possibles du niveau de production générale du systène. La prédation sélective des plus grands organismes par les poissons, classiquement décrite (BROOKS et DODSON, 1965 ; O'BRIEN, 1975), apparaît comme l'hypothèse la plus adéquate pour décrire l'ensemble du processus. Nos observations sont très comparables à celles de BROOKs et DODSON (1965) qui ont décrit les conséquences de l'introduction d'Alose sur le zooplancton d'un lac oligotrophe : réduction de la taille moyenne de 0,8 a $0,3 \mathrm{~mm}$, élimination des espèces de taille supérieure à 1 mon. A Sainte-Croix, suite au développement des ablettes, la taille moyenne du zooplancton est passée de 1,0 ma à $0,6 \mathrm{~mm}$ avec 82 des organismes entre 0,2 et $0,8 \mathrm{mun}$. En comparaison, la taille moyenne actuelle reste encore relativement importante. D. longispina, espece dominante en 1977-1978, n'est pas encore éliminèe du milieu après 10 ans. L"évolution de sa densité depuis juin 1982 montre cependant un déclin progressif des effectifs.

Autrefois dominante tout au long du cycle, cette espèce n'abonde plus qu'au printemps (avril-mai) (figure 5). Sa densité décroît dès juin. D'après ce schêma de distribution temporelle, il semblerait que le naintien de l'espêce soit réalisé grâce à l'existence de périodes "refuges". En effet, les mois d'avril-mai représentent une péxiode où : a) le phytoplancton est abondant en zone pélagique (bloom de phytoflagellés) et b) les ablettes sont retenues sur leur lieu de frai, la ponte ayant lieu vers la mi-naj (CHAPpaz et al., 1987). Durant cette période, seule une petite espèce de Bosmine est également abondante (B. longirostris ou B. coregonil. 
$10^{3}$ ind. $/ \mathrm{m}^{3}$

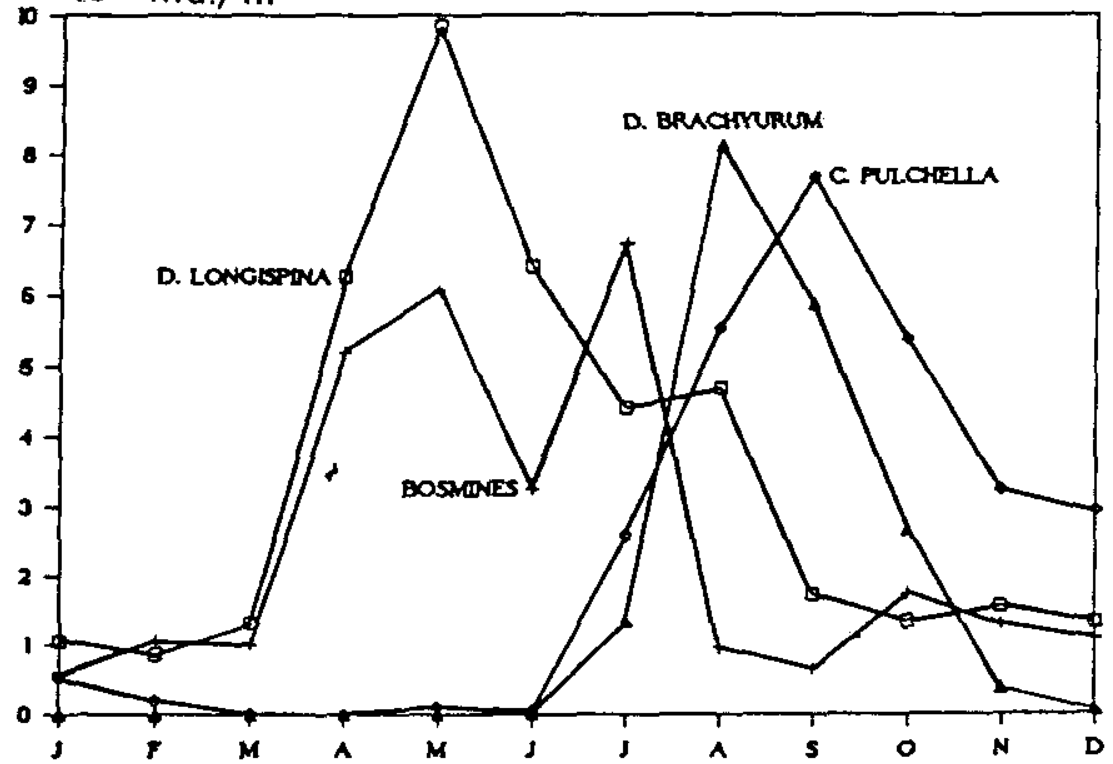

Figure 5. - Densités mensuelles moyennes des cladocêres (de juin 1982 a janviex 1983).

Figure 5. - Mean monthly density (ind. $\mathrm{m}^{3}$ ) of cladoceran species (from june 1982 to january 1987).

Durant la période de stratification proprenent dite, 0 . bhachyurum et $C$. pulchella, toutes deux uniquenent présentes de juillet à décembre, regroupent 1 'essentiel des effectifs. 0 . brachyurum, totalement absente en 1976, domine actuellement en août-septembre. De plus petite taille que 0 . longispina, elle est également moins vulnérable grâce à son aptitude à effectuer des mouvements imprévisibles (WRIGHT et O'BRIEN, 1984). Sa capacité de fuite est également plus forte lors de la phase d'aspiration de l'eau péribuccale par le préđateux (DRENNER et al., 1978). Il reste néanmoins que, depuis 1983, ses effectifs décroissent régulièrement alors que ceux de $C$. pulchella se maintiennent globalement tout come pour les bosmines. Durant cette deuxième moitié de l'année, on retrouve là aussi une association de deux espèces de tailles inégales pouvant tendre à rêduire la compétition interspécifique.

On peut également noter la réduction des effectifs des deux cyclopides de taille voisine de $1,5 \mathrm{~mm}$ (Macrocyclops albidus, Cyclops vicinus vicinusl alors que ceux du rotifère Asplanchna priodonta sont stables.

Ces diffërences d'évolution pluriannuelle entre les effectifs des grandes $(0,8$ a $1,6 \mathrm{~mm}$ ) et petite forme (environ $0,5 \mathrm{~mm}$ ) ne semblent pas pouvoir être expliquées par une modification des ressources trophiques. En effet, les bionasses et la composition spécifique du phytoplancton sont restées globalement inchangées de 1982 à 1986 avec une large dominance du nannophytoplancton $k 15$ un : flagellés, diatomées centriques du genre Cyclotella et des teneurs moyennes en chlorophylle a dans la zone photique comprises entre 0,5 et $2,5 \mathrm{mg} / \mathrm{m}^{3}$ (BIN-MOLE, 1987 ; EL HAFA, 1987). 
A la différence de nombreux systèmes prédateur-proies, le système observé à Sainte-Croix ne semble pas tendre vers un état proche de la stabilité en terme d'effectifs pour la proie principale (cf. 0 . longispina) et son prêdateur (1'Ablette). Ce cas est fréquent en limnologie où il est souvent décrit des prédateurs amenant leurs proies à l'extinction (MURDOCH et BENCE, 1987). Ces derniers auteurs expliquent notament ce résultat par une relative densité-indépendance du prédateur vis-à-vis de la densité de population de ses proies dans la mesure où : a) le caractère omnivore de la plupart des poissons leur permet de supplêer sans domage à une réduction temporaire (saisonnalitê) ou à plus long terme des effectifs de la proie, b) la longévitè des prédateurs adultes, très supérieure à celle des proies, a un effet déstabilisant sur le systène, c) les variations de densité de la totalité des proies disponibles ne se transmettent au prédateur qu'après un délaj très long en raison des possibilitês de stockage énergétique (graisses).

A Sainte-Croix, CHAPPAz et al. (1987) ont mis en évidence l'opportunisme de l'Ablette. Maximal pour les poissons pêchés en zone pélagique, le caractère zooplanctonophage du régime alimentaire s'atténue relativement en zone littorale (prêsence de mollusques) pour s'estomper totalement chez les ablettes capturées en amont, au débouché de la rivière (ingestion des macroinvertébrés en dérive). Cette opportunisme permettrait à l'Ablette de suppléer à la réduction progressive de la densité des populations de grandes formes zooplanctoniques tout en maintenant sur ces dernières une forte pression de prédation. Le caractère limitant de la ressource nutritionnelle essentielle (faibles densités de zooplancton : en moyenne 11000 ind. $/ \mathrm{m}^{3}$ ) entraine cependant une croissance lente et une faible fécondité de 1 'Ablette.

En définitive, l'examen de I'ensemble des composantes intervenant dans ce systême prédateur-proies démontre le caractère évolutif de la situation actuelle. En 1 'absence de toute modification majeure faugmentation des apports en provenance du bassin versant), la situation actuelle pourrait rapidement évoluer vers une raréfaction des grands cladocères (D. Longispina puis $D$. brachyurum\} et vers un zooplancton dominé par les rotifères, les deux espèces de Bosmines et $C$. pulchella. cependant, différents processus pourraient conduire vers un équilibre ne supposant pas une modification radicale du cortège faunistique :

- Une phase de croissance des populations de D. longispina limitêe au début de la période vernale lorsque la plupart des prédateurs sont sur les lieux de ponte.

- Le développenent de stratégies adaptatives chez les espèces proies tendant ã favoriser l'occupation de zones refuges (O' BRIEN, 1987). Les plus grands individus d'une espèce effectuent parfois les migrations verticales les plus importantes (ZARET et SUFFERN, 1976). A Sainte-Croix, des résultats préliminaires mettent en évidence une situation de ce type : les grandes écophases de $D$. longispina se maintiennent, de jour, à plus de $20 \mathrm{~m}$ de profondeur, contre 10-20 m pour les stades de développement plus jeunes (résultats non publiés).

- Une réduction de la pression de prédation pourrait intervenir en réponse à : a) une accentuation de la compétition interspécifique dans les zones littorales (accroissement des effectifs de perches juvéniles et d'alevins de gardons notamment), b) le développement d'espèces carnassières pour lesquel.les l'Ablette fournirait un excellent poisson fourrage. Depuis quelques années, en effet, la reproduction du brochet serible s'effectuer avec succès dans la retenue. 
Maillon intermédiaire de la chaîne trophique, la structure du peuplement zooplanctonique apparait ainsi conne un révélateur du mode de

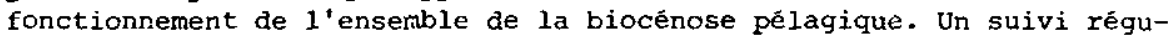
lier des peuplenents zooplanctonique et piscicole dans les années à venir serait d'un grand intérêt et permettrait de répondre à ces différentes questions.

\section{REMERCIEMENTS}

Ce travail a été réalisé dans le cadre des activités du GRECO C.N.R.S. n 71 "Fonctionnement des écosystèmes lacustres" et avec l'appui financier du PIREN-EAU, du CNRS et du Conseil Régional de Provence Côte d'Azur.

\section{RÉFÉRENCES BIBLIOGRAPHIQUES}

AMRANI J. (1987). Organisation sjatiotemporelle du avoplancton du lac de barrage de Sainte-Croiz (Provence, France). Thèse de Jème cycle, Université d'Aix-Marseille I, $148 \mathrm{pp}$.

BALVAY G. (1985). Structure et fonctionnement du réseau trophique dans les retenues artificielles. In Gestion piscicole des lacs et retenues artifisielles. Gerdeaux D. Billard R. Editeurs. INRA, Paris, 39-66.

BIN-HOLE R. (1987). Etude écologique du peuplement phytoplanctonique du lac de barrage de Sainte-Croix (Var). These de 3àme cycle, Université d'Aix-Marseille I, $158 \mathrm{pp}$.

BRESSAC Y. (1983). Recherches sur le peuplement zooplanctonique $d^{\prime}$ un lac de barrage : la retenue de Serxe-Poncon. Interprétation du cycle de Daphnia longispina. o.F. Mullex (Crustace, Cladocere). Rev. Er. Sc. Ear, 2 : 173-188.

BRESSAC Y. (1985). Le zooplanctor des lacs de barrage. Les retenues du sudEst de la Erance. Influence des irrivées d'eau et de la qualité du sestor sur la structure spatiaie du peuplement. These de Doctorat, Université d'Aix-Marseille I, $144 \mathrm{pp}$.

BRESSAC Y. CHAMPEAU A. (1985). Contribution l'etude du réseau trophique des lacs de barrage : le zooplancton des retenues de serre-Ponçon et sainte-croix. In Gestion piscicoie des lacs et retenues artificielles. Gerdeaux D. \& BILLARO R. Editeurs. INRA, Paris, 67-77.
BROOKS J., DODSON I. (1965). Predation, body size and composition of plankton. Science, $150: 28-35$.

CASPER S.J. (1985). Lake Stechlin: a temperate oligotrophic lake (Editeur). ionographiae Biologicae, 58 : 1-553.

CHAMPEAU A., GREGOIRE A., BRUN G. (1979). Le peuplement piscicole des lacs artificiels du verdon. Ann. Limnol., 14 : 245271.

ChAMpead A. (et collaborateurs) (1982). Les retenues hydroelectriques du verdon. Impact sur la riviere. Conséquences du narnage. Bull. Ecol., 13 (2) : 203-239.

Chappaz R. (1986). Etude piscicole de la retenue de Saint-Croir-Fontaine $t$ "Jvêque. Thèse de 3ène cycle, Universite d'aixMarseille I, 183 pp.

CHAPPAZ R., BRUN G, OLIVARI 6. (1987). Mise en èvidence de différences de régime alimentaire dans une population d'xblettes Neburrus Mlournus (L.) dans le lac de Sainte-Croix. Conséquences sur la croissance et la fécondité. Ann. Émnol., $23: 245-252$.

COOPER S.D., SMITH D.W., BENCE J.R. (1985) . Prey selection by freshwater predators with different foraging strategies. Can. j. Fish. Aquat. Sci., $42: 1720-1732$.

C.T.G.R.E.F. (1977). Inventaire piscicole de la retenue de Sainte-Croix-Fontaine l'Evèque. Ministère de l'Agriculture. Groupement $d^{\prime} A i=-$ Marseille, Doc. Pol., $52 \mathrm{pp}$. 
DRENNER R.W., STRICKLER J.R., O'BRIEN W.J. (1978). Capture probability : the role of zooplankter escape in the selective feeding of planktivorous fish. $t$. Fish. Res. Bd. Can., 35 : 1370-1373.

EL HAFA M. (1987). Bilan des apports de seis nutritifs au lac de Sainte-Croiz (Provence) et leur utilisation par le phytoplancion de la retenue. Thise de 3ène cycle, Universitê d'Aix-Marseille I, $130 \mathrm{pp}$.

FRYER G. (1957). The food of some freshwater cyclopolds copepods. Proc, Zool. Soc. London, 126 : 1-25.

GRECOIRE A. (1982). Contribution a l'etude hydrobiologique d'une rivière anénagée : le Verdon. Les lacs de barrage et les troncons de cours d'eau a debit regule. Cahier du Laboratoire d'yydrobiologie de Montereau, 13 : 1172 .

GRYGIEREX A. - HILLBRICHT-ILKOWSKA A., SPODNIEWSKA I. (2966). The effect of fish on plankton coumunity in ponds. Vehr. Int. Ver. Limmol., 16 : 13591366.

HRBACEX J., DVORAKONA M., KORINEK V. PROCHAzKOVA L. (1961). Demonstration of the effect of the fish stock on the species composition of zooplankton and the intensity of metabolism of the whole plankton assoclation. Vehr. Int. Ver. Limnol., 14 : 192-195.

HUTCHINSON G.E. (1967). A treatise on iimnology. II. Introduction to lake biology and the Limnoplankton. John Wiley. N.Y. 1115 PP.

JANSSEN J. (1980). Alewives (Alosa pseudohatengus) and Ciscoes (Coregonus artedii) as selective and non-selective planktivores. In : KERFOOT W.C. (Ed.), Evolution and ecology of rooplankton commonities. University press of New England, 580-586.

KERFOOT W.C., KELLOGG D.L. JY:, STRICKLER J.R. (1980). Visual observations of live zooplankters : evasion, escape and chemical defenses. In : KERFOOT w.c. (Ed.). Evolution and ecology of zoopiankton cormunities. University press of New England, 10-27.
DACT'E i., BOURLIERE F. (1971). ''́chantilionnage des peuplements animain des Tilieux aquatiques. Masson, Paris, 253 op.

LAZARRO X. (1987). A review of planktivorous fishes : Their evolution, feeding behaviours, selectivities, and insacts. تydrobiologia, 146 : 97-167.

MURDOCH พ.พ., BENCE J. (1987), Geteral predators and unstable prey populations. - $n$ : KERFOOT W.C. \& SIH A. (Eds.), Preadation. Direct and indirect impacs son zquatic commonities. University Press of New England, 17-30.

O'BRIEN W.J. (1975). SOme aspect of the Iimnology of the ponds and lakes of the Noatak drainage basin. Alaska. Veinr. Int. 7er. Limmol., $19: 472-479$.

O'BRIEN W.J. (1979). The predator-prey interaction of planktivorous fish and zooslankton. American Scientist., 67 :

572-581.

O'BRIEN W.J. (1987). Planktivory by freshwater fish : thrust and parry in the pelagia. In : KERFOOT W.C. \& SIH A. (Eds.), Ireadation. Direct and Indirect i-pacts on aquatic communities, University Press of New England, 3-16.

POURRIOT R. (1982). Ecologie du zinncton ies eaux continentales. Masson, Paris. $198 \mathrm{pp}$.

ZOURRIOT R. (1983). Influence sélective de la prédation sur la structure e dynamique du zooplancton d'eau douce. icta oecologica, Oecologie generci:s, 4 : i3 -25 .

:NRIGHT D.I., O'BRIEN W.J. (1984) . Differential location of Chroborus larice and Daphnia by fish : the importance of motion and visible size. Am. Midlancia Maturalist, $108: 68-73$.

ZARET T.M., SUFPERN J.S. (1976), Vertical tigration in zooplankton as a prectator avoidance manism. Limmol. Ocecrogr., 21 : $804-813$. 